\title{
Skilled Reach Training Induces Synaptogenesis on the Hippocampus after Left Hemorrhagic Stroke in Rats
}

\author{
Hyunmo Koo, $\mathrm{PhD}, \mathrm{PT}^{1)}$, MinheE KIM, $\mathrm{PhD}, \mathrm{PT}^{2)}$, \\ SunMin LeE, PhD, $\mathrm{PT}^{3)}$, JinTAE Han, PhD, $\mathrm{PT}^{1)}$ \\ 1) Department of Physical Therapy, College of Science, Kyungsung University: \\ 309 Suyeong-ro (Daeyeon-dong), Nam-gu, Busan, 608-736, Republic of Korea. \\ TEL:+82 51-663-4871,FAX: +82 51-663-4089,E-mail: jthan2001@ks.ac.kr \\ 2) Laboratory of Neuroscience, Department of Physical Therapy, College of Rehabilitation Science, \\ Daegu University \\ 3) Department of Occupational Therapy, Dongju College
}

\begin{abstract}
Purpose] The present study investigated the effect of upper limb skilled reach training in an experimental rat model of intracerebral hemorrhage (ICH). [Subjects and Methods] Forty five male Sprague-Dawley rats were subjected to ICH. Then, animals were randomly selected for the control (CON), ipsilateral (IST), and contralateral (CST) groups and skilled reach training was conducted for 2 or 4 weeks after brain injury. Motor behavioral tests, immunohistochemistry, western blotting, and 2,3,5-triphenyltetrazolium chloride staining analysis for evaluation of growth associated protein (GAP)-43 was used to assess synaptic plasticity and cognitive function. In addition, synaptic plasticity-related mRNA expressions were detected via RT-PCR. [Results] ICH-induced decrease of GAP-43 protein and synaptic plasticity-related mRNA expressions were recovered by skilled reach training in the IST and CST groups. Moreover, decreased ischemic volume and progressive neurobehavioral outcomes were observed in both the IST and CST groups. [Conclusion] In the rat model of ICH, skilled reach training promoted synaptic plasticity and cognitive function via increased GAP-43 protein and synaptic plasticity-related mRNA expressions.
\end{abstract}

Key words: Intracerebral hemorrhage, Skilled reach training, Synaptogenesis

(This article was submitted Aug. 25, 2011, and was accepted Sep. 28, 2011)

\section{INTRODUCTION}

Although stroke mortality seems to be declining and stroke incidence has recently been reported as stable, stroke involves severe impairment, including neurological deficits, sensory loss, hemiparesis, hemianopsia, and cognitive impairment in up to $50 \%$ of survivors ${ }^{1,2)}$. Stroke inducedcognitive impairments occur in the brain areas of memory, language, and judgment ${ }^{3}$. These cognitive impairments contribute to poor rehabilitation outcome, reduced ability in daily living, and lower capacity for work, as well as poor quality of life and increased burden on patients' families ${ }^{4,5)}$. Hence, it is important to tackle cognitive rehabilitation in patients with stroke, for return to work and home.

Rehabilitative cognitive training, serving the conditions and factors selected from all possible strategies such as motor ability and skilled learning, attempts to alter behavior and structural events after damage ${ }^{6}$. For confirmation of the effect of this clinical approach, neuro-rehabilitive strategies developed in animal models seem likely to be useful tools for researching the brain damage caused by cerebrovascular ischemia ${ }^{2}$. In particular, skilled reach movement training is one of the treatment methods for cognitive rehabilitation. This training involves putting a paw through a narrow opening to grasp small food pellets without dropping them, and requires comprehension of several degrees of freedom in the motor apparatus, a preferred trajectory and inter-joint coordination leading to the goal ${ }^{7,8)}$. Thus, the skilled reach movement training component of animal behavior in complex environments is essential for increasing dendritic plasticity and synaptogenesis, by modifying the neuronal growth-associated and synaptogenesis-related proteins, including GAP-43, as previously suggested ${ }^{8,9)}$. Nevertheless, the effect of skilled movement training needs better evidence before it can be used for patients with stroke. Therefore, the purpose of the present study was to investigate the effect of upper limb skilled reach training on both synapse formation and cognitive function via the expression increase of GAP-43 as well as Munc-18, SNAP-25, and Synapsin I in an experimental intracerebral hemorrhagic (ICH) rat model.

\section{SUBJECTS AND METHODS}

Forty five male Sprague-Dawley rats, weighing between $250 \mathrm{~g}$ and $300 \mathrm{~g}$, were used in the experiment. They were 
kept light/dark cycle with ad libitum access to food and water. All experiments were performed in accordance with protocols approved by the Animal Experiment Committee of University of Daegu, based on the NIH Guidelines for the Care and Use of Laboratory Animals (NIH publication, 1996). The animals were randomly divided into 3 groups: the control (CON), ipsilateral (IST), and contralateral (CST) skilled reach training groups. Rats were anesthetized by intraperitoneal injection with a mixture of $2 \mathrm{ml} / \mathrm{kg} 50 \%$ zoletil and 50\% xylazine hydrochloride, placed in stereotaxic frame and brain injury was induced, as previously described $^{10)}$. Animals were subjected to a $3 \mathrm{~mm}$ diameter craniotomy centered on the left side, $3.0 \mathrm{~mm}$ lateral to the sagittal suture and $0.2 \mathrm{~mm}$ posterior to the bregma, and a 30 -gauge Hamilton syringe needle was inserted through the hole into the striatum $6.0 \mathrm{~mm}$ ventrally. Then $1 \mu \mathrm{l}$ of a solution containing 0.23 collagen digestion unit of collagenase type VII (Sigma, St. Louis, MO, USA) was administered for $5 \mathrm{~min}$ to induce the intracerebral hemorrhagic injury. After collagenase injection, the wound was closed with sutures and the rat was given rest to recover. The total time of the surgical procedure was $10 \mathrm{~min}$.

Skilled training was conducted in a Plexiglas chamber (45 cm in height, $15 \mathrm{~cm}$ in width, $40 \mathrm{~cm}$ in length) containing a $1 \mathrm{~cm}$ by $10 \mathrm{~cm}$ window in the front wall, which allowed one of the rat's paw to reach through for a pellet. After being acclimated to the chamber with $4.5 \mathrm{mg}$ of sugar-flavored food pellets (Research Diets, New Brunswich, NJ, USA) put on a shelf in a small indentation $3 \mathrm{~cm}$ from the inside wall of the chamber, the animals were trained to reach for food in 15 min sessions, conducted 6 days per week for 2 or 4 weeks, as described in previous reports ${ }^{11,12)}$. The skilled reach training focused on the left anterior paw which was on the same side as the injured hemisphere in the ISP group, and on the right paw in the CST group. The CON group was not given any training after ICH injury. Thus, for the IST and CST groups, the pellets were aligned to either the left or the right side of the window. After a pellet was removed by a rat, the shelf was refilled continuously for the whole duration of the 15-minute experiment.

For sacrificed the animals were anesthetized with a mixture of $2 \mathrm{ml} / \mathrm{kg} 50 \%$ zoletil and $50 \%$ xylazine hydrochloride and perfused through the heart with $200 \mathrm{ml}$ of $0.9 \% \mathrm{NaCl}$ solution, followed by $4 \%$ paraformaldehyde solution. Their brains were removed, maintained in postfixative overnight, and then sectioned to a thickness of 30 $\mu \mathrm{m}$ for immunohistochemistry to examine the protein expression for synaptogenesis the brain tissue. In brief, the sections were washed $(3 \times 10 \mathrm{~min})$ in $0.01 \mathrm{M}$ phosphatebuffered saline solution (PBS; $\mathrm{pH}$ 7.2) and incubated with appropriate primary antibodies for $12 \mathrm{~h}$ at room temperature. The antibody was diluted to 1:200 with a solution of Triton X-100 and normal donkey serum. After incubation in primary antibody, the sections were rinsed $(3 \times 10 \mathrm{~min})$ in PBS, incubated for $90 \mathrm{~min}$ at room temperature with antimouse Immunoglobulin (Ig) G (Vector Laboratories Inc, USA), diluted 1:25 with a solution of Triton X-100 and normal donkey serum. After incubation in secondary antibody, the sections were rinsed $(3 \times 10 \mathrm{~min})$ in PBS, and incubated for $1 \mathrm{~h}$ at room temperature with a Vectastain Elite ABC-kit (Vector Laboratories Inc, USA). Then, the sections were rinsed again with PBS and incubated for 10 min in $0.04 \mathrm{mg}$ of 3,3'-diaminobenzidine (DAB) in $200 \mathrm{ml}$ distilled water. The sections were then incubated for 1 min in DAB solution with $35 \% \mathrm{H}_{2} \mathrm{O}_{2}$. The DAB sections were rinsed again with $\mathrm{PBS}(3 \times 10 \mathrm{~min})$ to halt the chromagen reaction, wet-mounted on gelatin/chromiumcoated slides, and allowed to air-dry overnight. The sections were then dehydrated through a series of alcohols, soaked in xylene, and cover-slipped with Clarion (Biomedia, USA).

To perform the western blotting analysis, for quantitative investigation of the protein expression for synaptogenesis in the brain tissue, the brains from each group of rats were collected, washed twice in PBS, and then homogenized and lysated with buffer $\left(137 \mathrm{mM} \mathrm{NaCl}, 8.1 \mathrm{mM} \mathrm{Na} 2 \mathrm{HPO}_{4}, 2.7\right.$ $\mathrm{mM} \mathrm{KCl}, 1.5 \mathrm{mM} \mathrm{KH}_{2} \mathrm{PO}_{4}, 2.5 \mathrm{mM}$ EDTA, $1 \mathrm{mM}$ dithiothreitol, $0.1 \mathrm{mM}$ PMSF, and $10 \mu \mathrm{g} / \mathrm{ml}$ leupeptin [pH 7.5]) for $30 \mathrm{~min}$ on ice. Then, the lysates were centrifuged for 10 min at $15,000 \mathrm{rpm}$ at $4{ }^{\circ} \mathrm{C}$, and the protein concentration was determined, as described previously ${ }^{13}$. Equal amounts of protein (i.e. $40 \mu \mathrm{g}$ ) were resolved via $10 \%$ sodium dodecyl sulfate-polyacrylamide gel electrophoresis (SDS-PAGE) and transferred to nitrocellulose membranes. The blots were then washed with TBST $(10 \mathrm{mM}$ Tris $\cdot \mathrm{HCl}$ [pH 7.6], $150 \mathrm{mM} \mathrm{NaCl}, 0.05 \%$ Tween 20), blocked with $5 \%$ skim milk for $1 \mathrm{~h}$, then incubated with the appropriate primary antibodies at the dilutions recommended by the suppliers. The membranes were washed, and the primary antibodies were detected using horseradish peroxidaseconjugated goat anti-rabbit IgG or goat-anti mouse IgG. The bands were then visualized via enhanced chemiluminescence (Amersham Pharmacia Biotech, Piscataway, NJ, USA). Immunohistochemistry and immunoblotting of GAP-43 expression were performed for each group. The values relative to the CON group in the left hemisphere, were compared between groups and sides at 4 weeks.

The reverse transcription-polymerase chain reaction (RT-PCR) was used to evaluate the mRNA expression for synaptogenesis in the brain tissue. Total RNA was acquired from the collected brains using STAT-60, a monophasic solution of phenol and guanidine isothiocyanate (Tel-Test, Friendswood, TX, USA). Reverse transcription (RT) was conducted using $3 \mu \mathrm{g}$ of RNA with a reverse transcription system kit (AccuPower RT PreMix, Bioneer, Daejeon, Korea) with oligo(dT) ${ }_{18}$ primers. Five microliters of the RT products were then amplified with a polymerase chain reaction (PCR) kit (AccuPower PCR Premix) under the following conditions: denaturation at $94^{\circ} \mathrm{C}$ for $5 \mathrm{~min}$ and 30 cycles at $94^{\circ} \mathrm{C}$ for $45 \mathrm{sec}, 55^{\circ} \mathrm{C}$ for $30 \mathrm{sec}$, and $72^{\circ} \mathrm{C}$ for 30 sec, followed by $5 \mathrm{~min}$ of extension at $72^{\circ} \mathrm{C}$. The primers used were: 5'-CTGTGTGCCACTCTTCAGGA-3' (forward), 5'-AGGTATGCACCC-AGAGTGATGC-3 (reverse) for Munc-18; 5'-CCTCCACTCTTGCTACCTGC-3'(forward), 5' -TCCTCTGCATCTCCTCCAGT-3' (reverse) for SNAP-25; and 5'-CATGGCACGTAATGGAGACTACCGCA-3' (forward), 5'-CCGCCAGCATGCCTTC3 '(reverse) for Synapsin-1. PCR for $\beta$-actin was also conducted as an RNA quantity control.

To confirm the area of brain injury, after the animals 
were anesthetized and sacrificed, their brains were extracted, immersed for $10 \mathrm{~min}$ in cold PBS, and sliced into $2.0 \mathrm{~mm}$ thick sections. The sliced brain samples were incubated in 2\% 2,3,5-triphenyltetrazolium chloride (TTC) (Sigma-Aldrich, USA), dissolved in PBS for $20 \mathrm{~min}$ at $37^{\circ} \mathrm{C}$, and subsequently transferred to $4 \%$ parafomaldehyde solution for fixation. Brain damage was revealed by a lack of TTC staining, which indicates that the tissues were dehydrogenase-deficient. The volume of brain damage was measured in each slice and the injured area was determined as a percentage of total brain area.

To confirm the histological changes in brain tissue, the sections were washed $(3 \times 10 \mathrm{~min})$ in $0.01 \mathrm{M}$ phosphatebuffered saline (PBS, pH 7.2). Then, they were dipped in hematoxylin solution for $5 \mathrm{~min}$ and washed in running tap water. The sections were dipped in $1 \% \mathrm{HCl}$-alcohol solution 4 times, then washed in running tap water for $5 \mathrm{~min}$. After washing, they were dipped in eosin solution for $2 \mathrm{~min}$, dehydrated via a graded series of alcohols, soaked in xylene and cover-slipped with Clarion (Biomedia, USA).

For the object-recognition test, two identical objects, heavy enough not to be moved by rats, were placed $10 \mathrm{~cm}$ from the corners of a box $(100 \mathrm{~cm}$ width, $100 \mathrm{~cm}$ length, $30 \mathrm{~cm}$ height). Rats were placed at the center of the box. A video camera was suspended above the box to record the rats' behavior. First, a rat was exposed to the objects for 3 min for a preliminary search. Then, the rat was placed back in the cage for $5 \mathrm{~min}$, and one of the objects was switched to a new object during the rest period. The rat was then placed in the box again for a 3 min search, as previously described $^{14,15)}$.

The object-location test was prepared and performed like the object-recognition test. It differs in that one of the objects is placed in a new location, instead of switching one of the objects to a new object. The time spent searching each object was measured, and the results of these tests were expressed as percentages of time spent searching for the object switched to a new object (in case of the objectrecognition test) or for the object moved to a new location (in case of the object-location test) per total time spent searching for both objects, as previously described ${ }^{14,15)}$.

The results are expressed as mean \pm standard error ( $\mathrm{S}$. E.). All results were analyzed with analysis of variance (ANOVA), and comparisons of the mean values between treatment and control groups were made using the
Bonferroni-Dunn test. Differences were considered statistically significant at $p<0.05$. All analyses were performed using SPSS for Windows (v. 12.0K, SPSS Inc., USA).

\section{RESULTS}

The ICH-induced reduction of GAP-43 expression increased significantly with skilled reach training in both the ipsilateral and contralateral limbs at 4 weeks $(p<0.05)$. A more significant increase in GAP-43 expression was found in the CST group than in the IST group. In particular, an increase was observed in the injured hemisphere of the CST group, compared to both hemispheres in the CON and IST groups $(\mathrm{p}<0.05)$. There was a significant difference between the left and right hemispheres only in the CST group $(\mathrm{p}<0.05)$ (Table 1$)$.

The ICH-induced decrease of Munc-18, SNAP-25, and Synapsin I, which are mRNAs related to synaptogenesis, were reversed in both hemispheres of the IST group and in the injured hemisphere of the CST group, compared to the CON group $(\mathrm{p}<0.05)$. There was a significant difference between the left and right hemisphere only in the CST group $(\mathrm{p}<0.05)$ (Table 2$)$.

To confirm the area of brain injured by ICH, TTC staining was performed. In the CST group, the ischemic area was less extended than in the CON group (Fig. 1A). Histology with H-E staining showed that the ischemic damage was greater and glial scar tissue with many inflammatory cells, heavily stressed by the ischemia, were predominantly observed in the injured area in the CON group. In contrast, only a small number of inflammatory cells were observed in the CST group (Fig. 1B).

Both the object-recognition and object-location tests were carried out to investigate the working memory elicited by skilled reach training after ICH as working memory is sensitive to alterations of cognitive function after brain damage. In the object-recognition test, the proportion of time spent of exploring tended to increase with lapse of time in all groups $(p<0.05)$. In particular, the increase was more significant in the CST group than in the CON group, based on the result at 4 weeks after lesion $(p<0.05)$. Similarly, in the object-location test, the proportion of time spent exploring also increased with the lapse of time in all groups $(p<0.05)$. Based on the result at 4 weeks, there was improvement of cognition in both the IST and CST groups,

Table 1. The effect of skilled reach training after ICH on the expression of GAP-43

\begin{tabular}{lcc}
\hline \multirow{2}{*}{ Group } & \multicolumn{2}{c}{ Relative optical density (\% of CON in Lt. hemisphere) } \\
\cline { 2 - 3 } & Left hemisphere & Right hemisphere \\
\hline CON & $100.3 \pm 2.3$ & $104.3 \pm 4.0$ \\
IST & $119.0 \pm 3.0^{*}$ & $108.7 \pm 4.3$ \\
CST & $150.2 \pm 7.3^{*}$ & $127.3 \pm 6.5^{*} \S$ \\
\hline
\end{tabular}

To confirm the expression of GAP-43, immunohistochemistry and western blotting analyses were conducted on the CON, IST, and CST groups. Each example shown is representative of three experiments. The optical density values are the mean \pm S.E. of the values of relative to the left hemisphere value of the CON group. They were determined by densitometry relative to $\beta$-actin, respectively. Statistical analysis was performed using one-way ANOVA and the independent t-test. $* \mathrm{p}<0.05$ versus $\mathrm{CON}$ group. $\S \mathrm{p}<0.05$ versus left hemisphere. 
Table 2. The effect of skilled reach training after ICH on the expression of Munc-18, SNAP-25, and Synapsin I mRNA

\begin{tabular}{|c|c|c|c|}
\hline \multirow{2}{*}{\multicolumn{2}{|c|}{ Group }} & \multicolumn{2}{|c|}{ Relative optical density ( $\%$ of CON in Lt. hemisphere) } \\
\hline & & Left hemisphere & Right hemisphere \\
\hline \multirow{3}{*}{ Munc-18 } & $\mathrm{CON}$ & $100.9 \pm 6.6$ & $101.5 \pm 2.9$ \\
\hline & IST & $118.6 \pm 3.4^{*}$ & $126.8 \pm 3.5^{*}$ \\
\hline & CST & $154.4 \pm 3.6^{*}$ & $98.1 \pm 6.3 \S$ \\
\hline \multirow{3}{*}{ SNAP-25 } & $\mathrm{CON}$ & $100.4 \pm 4.3$ & $103.3 \pm 2.0$ \\
\hline & IST & $123.5 \pm 2.8^{*}$ & $131.5 \pm 3.6^{*}$ \\
\hline & CST & $156.9 \pm 3.6^{*}$ & $99.0 \pm 3.0 \S$ \\
\hline \multirow{3}{*}{ Synapsin I } & $\mathrm{CON}$ & $100.8 \pm 4.3$ & $104.3 \pm 2.6$ \\
\hline & IST & $128.2 \pm 3.9^{*}$ & $123.7 \pm 5.3$ \\
\hline & CST & $162.7 \pm 3.4 *$ & $110.1 \pm 3.5 \S$ \\
\hline
\end{tabular}

To confirm the expression of Munc-18, SNAP-25, and Synapsin I mRNA, RT-PCR was conducted. The optical density values are the mean \pm S.E. of the relative to the left hemisphere value of the CON group. They were determined by densitometry relative to $\beta$-actin. Statistical analysis was performed using one-way ANOVA and the independent t-test.* $p<0.05$ versus CON group. § $\mathrm{p}<0.05$ versus left hemisphere.

A

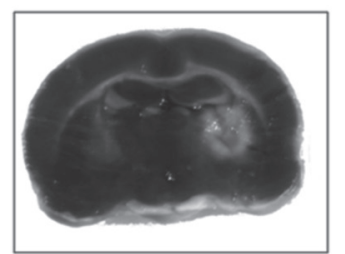

B

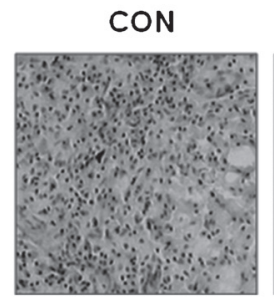

IST

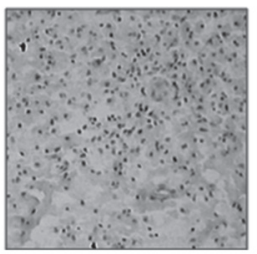

IST
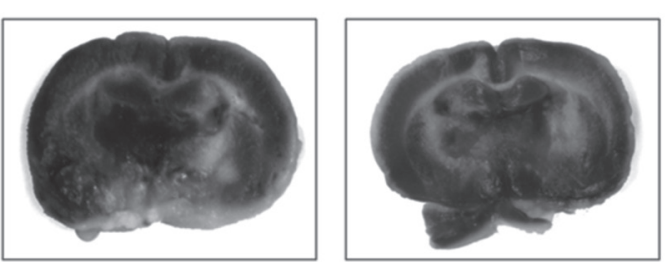

CST

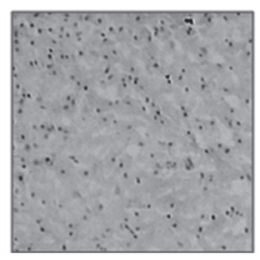

Fig. 1. The effect of skilled reach training after ICH on TTC and H\&E staining To confirm the ischemic volume of the brain-injured area, TTC (A) and H\&E staining (B) were conducted for the CON, IST, and CST groups.

compared to $\mathrm{CON}(\mathrm{p}<0.05)$ (Table $3 \mathrm{~A}, \mathrm{~B})$.

\section{DISCUSSION}

Stroke causes various problems for health-care, and rehabilitation occupies an important part of stroke patients' care. Hand and arm function therapy can improve activities of daily living; however, information and evidence about the clinical effect of various strategies of cognitive rehabilitation are scarce ${ }^{6}$. Rehabilitative approaches have recently adopted methods to induce adaptability and plasticity of the brain, after studies of responses to various training methods for expansion of motor leaning ${ }^{16)}$. The present study investigated the effect of skilled reach training on synaptic plasticity and cognitive function through increases in GAP-43 protein and synaptic plasticity-related mRNA expression in the rat model of ICH.

GAP-43 is the intracellular protein abundantly found in growing axon terminals of the guiding mechanism. It establishes and reorganizes synaptic connections, and has important roles in axonal growth, neurotransmitter release, and learning and memory ${ }^{17)}$. GAP-43 is expressed in sprouting axons of the adult CNS and in presynaptic nerve terminals ${ }^{18,19)}$. In the present results, skilled reach training increased GAP-43 expression after ICH. Moreover, it also increased the expression of synaptogenesis-related proteins, including Munc-18, SNAP-25, and Synapsin I. These are members of the terminal-specific phosphoproteins family involved in synaptic vesicle clustering and release ${ }^{20)}$. In addition, it has been reported that GAP-43 and Synapsin I appear to act in concert under the influence of activity defendant training ${ }^{17)}$. Skilled reach training also increased 
Table 3. The effect of skilled reach training after ICH on the object-recognition and object-location tests.

\begin{tabular}{lccc}
\hline A & \multicolumn{3}{c}{ \% Exploration time } \\
\hline \multirow{2}{*}{ Group } & Before & 2 weeks & 4 weeks \\
\cline { 2 - 4 } & $43.8 \pm 6.6$ & $51.20 \pm 5.4$ & $62.8 \pm 8.6^{*}$ \\
CON & $42.6 \pm 5.2$ & $57.00 \pm 11.4^{*}$ & $67.0 \pm 7.5^{*}$ \\
IST & $39.9 \pm 3.5$ & $60.10 \pm 9.6^{*}$ & $75.0 \pm 8.2^{*} \S$ \\
\hline
\end{tabular}

B

\begin{tabular}{lccc}
\hline \multirow{2}{*}{ Group } & \multicolumn{3}{c}{ \% Exploration time } \\
\cline { 2 - 4 } & Before & \multicolumn{1}{c}{ 2 weeks } & 4 weeks \\
\hline CON & $43.5 \pm 5.1$ & $56.20 \pm 7.5^{*}$ & $63.8 \pm 8.9^{*}$ \\
IST & $40.6 \pm 3.3$ & $59.10 \pm 10.6^{*}$ & $73.9 \pm 10.9 * \S$ \\
CST & $40.9 \pm 2.9$ & $65.10 \pm 8.4^{*}$ & $80.0 \pm 9.9^{*} \S$ \\
\hline
\end{tabular}

For cognitive improvement, object-recognition (A) and object-location (B) tests were carried out. Entries are the mean exploration ratio \pm S.E. ([time with new object / time with old + time with new object] $\times 100 \%)$ per group for the object-recognition and objectlocation tests. Statistical analysis was performed by using two-way ANOVA * $\mathrm{p}<0.05$ versus before training between the period; $\S \mathrm{p}<0.05$ versus $\mathrm{CON}$ between the group.

BDNF expression in another of our studies (unpublished data). It has been suggested that BDNF and Synapsin I levels are reduced after injury and the subsequent increase after training may arise from the cellular dynamics of synaptic plasticity and function ${ }^{21)}$.

In the present study, we found significant improvement of the cognitive function, as assessed by object recognition and location tests. This result is in line with results from previous studies showing that physical exercise-induced improvements in learning and memory are directly associated with increases in activity-dependent synaptic plasticity and altered gene expression ${ }^{22,23)}$. Such improvements have been found in the hippocampus, a highly plastic structure located in the medial temporal region of the brain, which is important for activity-dependent (i.e. working) learning and memory ${ }^{24)}$. Although various forms of hippocampal-dependent learning have been reported, the main effects of physical training on hippocampal plasticity are long-term potentiation (LTP, a well-recognized cellular analogue of learning and memory), and enhanced spatial learning ${ }^{25)}$.

Structural changes after brain lesions have been demonstrated to induce changes in forelimb function, as well as in neuronal growth-associated and synaptogenesis-related proteins. Lesions of the forelimb representation area of the sensory motor cortex result in impairment of use on the affected side, and patients generally rely increasingly on the unimpaired side ${ }^{26)}$. Examples of training approaches for patients with motor deficiencies after stroke include the motor relearning program and repetitive arm training, such as the constraint-induced movement therapy (CIMT) ${ }^{27,28)}$. In our study, skilled reach training on the more affected side, as seen in the CST group, may be comparable to CIMT. Moreover, in the present study, synaptic plasticity and cognitive function significantly increased in the CST group, as reported in previous studies 29,30 ).
Moreover, in the present study, the increase in also occurred in the IST group, when training was given to the less affected limb. Previous studies have reported dendritic changes are partially dependent on the use of the ipsilateral forelimb, and synaptogenesis can be enhanced by acrobatic training, a complex type of motor skill task ${ }^{8,9)}$. Therefore, the enhanced capacity to learn with the unimpaired forelimb might facilitate activity in the less affected hemisphere after training, and might be associated with recovered motor function and use-dependent brain plasticity ${ }^{31,32)}$. However, it might also exacerbate learned non-use of the impaired forelimb. These possibilities seem to warrant further investigations of the neural basis of this phenomenon.

The present results indicates that, in the rat model of $\mathrm{ICH}$, skilled reach training facilitated synaptic plasticity and cognitive function through increase of GAP-43 protein and synaptic plasticity-related mRNA expressions. Thus, the training component of animal behavior in complex environments appears to be essential for large increases in dendritic plasticity and synaptogenesis which are inducing by modification of synapse-saving or apoptosis-reducing molecular cascades of the injured area, as well as in remote indirectly injured brain regions ${ }^{8,9)}$. This connectivity in the nervous system indicates the basis of the complexity of neural circuits that support complex hand movements. These integrated circuits involve various cerebral cortical regions, the nature of which depends on the neural circumstances as well as environmental stimulation. Especially, environmental stimulation by external change is determined by functional performance which consists of task complexity, nature of concurrent processing, sensory triggers, and planning requirements ${ }^{33)}$.

\section{ACKNOWLEDGMENT}


This research was supported by Kyungsung University Research Grants in 2011.

\section{REFERECES}

1) Page SJ, Szaflarski JP, Eliassen JC, et al.: Cortical plasticity following motor skill learning during mental practice in stroke. Neurorehabil Neural Repair, 2009, 23: 382-388.

2) Cheatwood JL, Emerick AJ, Kartje GL: Neuronal plasticity and functional recovery after ischemic stroke. Top Stroke Rehabil, 2008, 15: 42-50.

3) McDonnell MN, Bryan J, Smith AE, et al.: Assessing cognitive impairment following stroke. J Clin Exp Neuropsychol, 33: 2011, 33: 1-9.

4) Hofgren C, Bjorkdahl A, Esbjornsson E, et al.: Recovery after stroke: cognition, ADL function and return to work. Acta Neurol Scand, 2007, 115: 73-80.

5) Hommel M, Miguel ST, Naegele B, et al.: Cognitive determinants of social functioning after a first ever mild to moderate stroke at vocational age. J Neurol Neurosurg Psychiatry, 2009, 80: 876-880.

6) Langhorne P, Bernhardt J, Kwakkel G: Stroke rehabilitation. Lancet, 2011, 377: $1693-1702$.

7) Cirstea MC, Mitnitski $\mathrm{AB}$, Feldman $\mathrm{AG}$, et al.: Interjoint coordination dynamics during reaching in stroke. Exp Brain Res, 2003, 151: 289-300.

8) Kleim JA, Jones TA, Schallert T: Motor enrichment and the induction of plasticity before or after brain injury. Neurochem Res, 2003, 28: 1757-1769.

9) Keyvani K, Schallert T: Plasticity-associated molecular and structural events in the injured brain. J Neuropathol Exp Neurol, 2002, 61: 831-840.

10) Park JW, Bang MS, Kwon BS, et al.: Early treadmill training promotes motor function after hemorrhagic stroke in rats. Neurosci Lett, 2010, 471: 104-108.

11) Girgis J, Merrett D, Kirkland S, et al.: Reaching training in rats with spinal cord injury promotes plasticity and task specific recovery. Brain, 2007, 130: 2993-3003.

12) Maldonado MA, Allred RP, Felthauser EL, et al.: Motor skill training, but not voluntary exercise, improves skilled reaching after unilateral ischemic lesions of the sensorimotor cortex in rats. Neurorehabil Neural Repair, 2008 , 22: 250-261.

13) Bradford MM: A rapid and sensitive method for the quantitation of microgram quantities of protein utilizing the principle of protein-dye binding. Anal Biochem, 1976, 72: 248-254.

14) Bisagno V, Ferguson D, Luine VN: Chronic D-amphetamine induces sexually dimorphic effects on locomotion, recognition memory, and brain monoamines. Pharmacol Biochem Behav, 2003, 74: 859-867.

15) Ennaceur A, Neave N, Aggleton JP: Spontaneous object recognition and object location memory in rats: the effects of lesions in the cingulate cortices, the medial prefrontal cortex, the cingulum bundle and the fornix. Exp Brain Res, 1997, 113: 509-519.

16) Dobkin BH: Strategies for stroke rehabilitation. Lancet Neurol, 2004, 3: 528-536.

17) Gomez-Pinilla F, Ying Z, Roy RR, et al.: Voluntary exercise induces a BDNF-mediated mechanism that promotes neuroplasticity. J Neurophysiol, 2002, 88: 2187-2195.
18) Chen B, Wang JF, Sun X, et al.: Regulation of GAP-43 expression by chronic desipramine treatment in rat cultured hippocampal cells. Biol Psychiatry, 2003, 53: 530-537.

19) Jutapakdeegul N, Afadlal S, Polaboon N, et al.: Repeated restraint stress and corticosterone injections during late pregnancy alter GAP-43 expression in the hippocampus and prefrontal cortex of rat pups. Int J Dev Neurosci, 2010, 28: 83-90.

20) Griesbach GS, Hovda DA, Molteni R, et al.: Voluntary exercise following traumatic brain injury: brain-derived neurotrophic factor upregulation and recovery of function. Neuroscience, 2004, 125: 129-139.

21) Ying Z, Roy RR, Edgerton VR, et al.: Exercise restores levels of neurotrophins and synaptic plasticity following spinal cord injury. Exp Neurol, 2005, 193: 411-419.

22) Farmer J, Zhao X, van Praag H, et al.: Effects of voluntary exercise on synaptic plasticity and gene expression in the dentate gyrus of adult male Sprague-Dawley rats in vivo. Neuroscience, 2004, 124: 71-79.

23) O'Callaghan RM, Ohle R, Kelly AM: The effects of forced exercise on hippocampal plasticity in the rat: A comparison of LTP, spatial- and non-spatial learning. Behav Brain Res, 2007, 176: 362-366.

24) Lynch MA: Long-term potentiation and memory. Physiol Rev, 2004, 84: 87-136.

25) Pan $X$, Tsukada M: A model of the hippocampal-cortical memory system. Biol Cybern, 2006, 95: 159-167.

26) Luke LM, Allred RP, Jones TA: Unilateral ischemic sensorimotor cortical damage induces contralesional synaptogenesis and enhances skilled reaching with the ipsilateral forelimb in adult male rats. Synapse, 2004, 54: 187-199.

27) Ertelt D, Small S, Solodkin A, et al.: Action observation has a positive impact on rehabilitation of motor deficits after stroke. Neuroimage, 2007, 36 Suppl 2: T164-T173.

28) Elbert T, Rockstroh B, Bulach D, et al.: New developments in stroke rehabilitation based on behavioral and neuroscientific principles: constraintinduced therapy. Nervenarzt, 2003, 74: 334-342.

29) Zhao C, Wang J, Zhao S, et al.: Constraint-induced movement therapy enhanced neurogenesis and behavioral recovery after stroke in adult rats. Tohoku J Exp Med, 2009, 218: 301-308.

30) Mestriner RG, Pagnussat AS, Boisserand LS, et al.: Skilled reaching training promotes astroglial changes and facilitated sensorimotor recovery after collagenase-induced intracerebral hemorrhage. Exp Neurol, 2011, 227: 53-61.

31) Johansen-Berg H, Rushworth MF, Bogdanovic MD, et al.: The role of ipsilateral premotor cortex in hand movement after stroke. Proc Natl Acad Sci U S A, 2002, 99: 14518-14523.

32) Lindberg PG, Schmitz C, Engardt M, et al.: Use-dependent up- and downregulation of sensorimotor brain circuits in stroke patients. Neurorehabil Neural Repair, 2007, 21: 315-326.

33) Small SL, Buccino G, Solodkin A: The mirror neuron system and treatment of stroke. Dev Psychobiol, 2010, [Epub ahead of print]. 